\title{
Acute Peritonitis Caused by a Fibrosarcoma of the Transverse Colon in an Adult
}

\author{
Seok Youn Lee, Jung Nam Kwon, Keun Young Kim ${ }^{1}$ \\ Department of Surgery, Wonkwang University Sanbon Hospital, Wonkwang University School of Medicine, Gunpo; ${ }^{1}$ Department of Surgery, \\ Wonkwang University Hospital, Wonkwang University School of Medicine, Iksan, Korea
}

\begin{abstract}
A fibrosarcoma is a malignant mesenchymal tumor derived from fibrous connective tissue. It usually develops in the deep soft tissues of the extremities, as well as the trunk, head, and neck. In extremely rare cases, a fibrosarcoma may occur in the gastrointestinal tract. Most cases of fibrosarcoma in the gastrointestinal tract have been observed in the pediatric age group while only a few cases have been reported in adults. A 61-year-old male presented with pain in the entire abdominal region. Chest radiography showed free air in the subphrenic space. After an emergency operation, we found a solid mass around the transverse colon and performed a segmental resection with a lymphatic dissection of the transverse colon, including the mass. A pathologic examination showed a fibrosarcoma with a perforation. There was no perioperative complication. The patient was discharged on postoperative day 11 and had follow-ups for 1 year without any recurrence.
\end{abstract}

Keywords: Fibrosarcoma; Transverse colon; Adult

\section{INTRODUCTION}

A fibrosarcoma is a malignant tumor arising from fibrous connective tissue. It is characterized by the presence of immature, proliferating fibroblasts or undifferentiated anaplastic spindle cells in a storiform pattern. It is most common in the extremities and trunk, occurring less frequently in the head and neck and very rarely in the alimentary tract. Furthermore, a fibrosarcoma is most frequently observed in the early years of life and is extremely rare in adults. Review articles have reported only four cases involving a fibrosarcoma of the colon [1-4]. Here, we report our experience with a case of acute peritonitis caused by a fibrosarcoma of the transverse colon in an adult.

Received: August 2, 2014 - Accepted: November 19, 2014

Correspondence to: Seok Youn Lee, M.D.

Department of Surgery, Wonkwang University Sanbon Hospital, Wonkwang

University School of Medicine, 327 Sanbon-ro, Gunpo 435-040, Korea

Tel: +82-31-390-2218, FAX:+82-31-390-2983

E-mail: sylee314@hotmail.com

(C) 2014 The Korean Society of Coloproctology

This is an open-access article distributed under the terms of the Creative Commons Attribution NonCommercial License (http://creativecommons.org/licenses/by-nc/3.0) which permits unrestricted non-

commercial use, distribution, and reproduction in any medium, provided the original work is properly cited.

\section{CASE REPORT}

A 61-year-old male who presented with complaints of abdominal pain and nausea was admitted to the Emergency Department. The patient had no previous history of disease or abdominal trauma. In cardiopulmonary auscultation, nothing unusual was detected. At the time of admission, the blood pressure, temperature, and pulse were $120 / 70 \mathrm{mmHg}, 38.3^{\circ} \mathrm{C}$, and 80 beats per minute, respectively. However, the patient was very ill-looking. On physical examination, rebound tenderness and muscle rigidity were elicited in the upper abdomen.

The peripheral blood test had a leukocyte count of $13,500 / \mathrm{mm}^{3}$, a hemoglobin count of $13 \mathrm{~g} / \mathrm{dL}$, and a platelet count of 335,000/ $\mathrm{mm}^{3}$. Coagulation studies were normal. Electrolyte, biochemical, and urine analyses were within normal ranges. The chest radiograph revealed free air in the subphrenic space (Fig. 1). Computed tomography (CT) of the abdomen revealed the presence of a largemass lesion. This massive lesion had a wall thickening which was a luminal obstruction in the transverse colon (maximal diameter, $5.3 \mathrm{~cm}$ ) (Fig. 2). No ascites were found. We initially diagnosed the patient as having peritonitis that caused a malignant obstruction in the transverse colon, and we performed an emergency operation. A turbid fluid was encountered upon entry into the peritoneal cavity. A round mass, about $5 \mathrm{~cm}$ in diameter, was observed 
Volume 30, Number 6, 2014

in the left upper abdominal cavity, and it seemed to be wrapped with the omentum around a distal part of the transverse colon. Mild dilatation of the colon proximal to the lesion was found. A segmental resection with a lymphatic dissection of the transverse

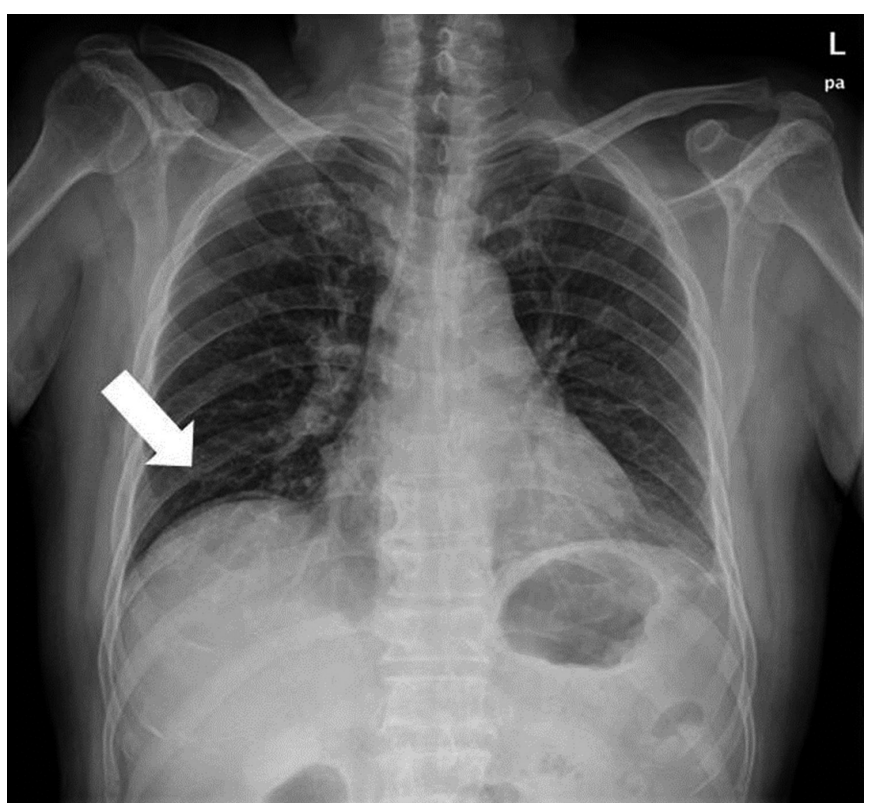

Fig. 1. Chest radiograph shows free air in the subphrenic space (arrow).
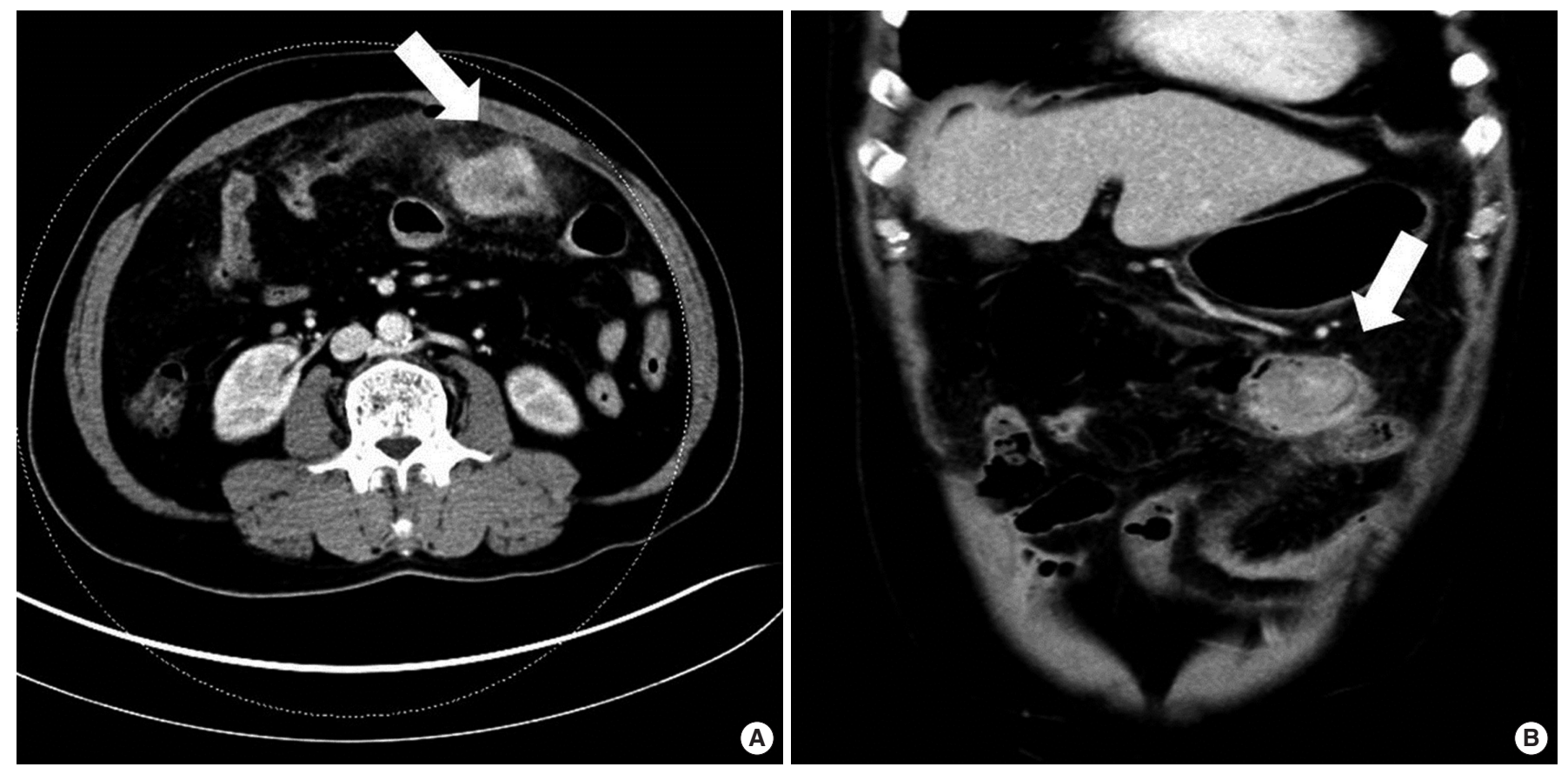

Fig. 2. Abdomen-pelvic computed tomography scan shows an irregular heterogeneous wall thickening and inflammatory changes in the transverse colon with pericolic fat infiltration (arrows): (A) axial view and (B) coronal view. ileostomy was made.

A large perforation site was visible on the colonic surface. A cut section revealed a yellowish, firm lesion that encircled the fibroadipose tissue (Fig. 3). No lymph node enlargement or other unusual pathological findings were found during surgery. At postoperative day 11, the patient was discharged without any complications. Microscopically, a tumor that arose from the wall of the colon was found; this tumor had eroded the mucosa and extended into the mesocolon. The architecture of the tumor consisted of interlacing bundles of fibrillar collagen that was lined by cells with spindle and stellate nuclei. The nuclei had a few mitoses. The immunohistochemical stain of the tumor was positive for vimentin and negative for smooth muscle actin (SMA), c-kit, CD31, CD34, Pan CK, Desmin, and al-antichymotrypsin (Fig. 4). The pathologic analysis report described a fibrosarcoma. Because complete surgical resection was performed and the surgical resection margins were free of tumor, the patient was observed without adjuvant therapy. One year postoperatively, radiologic evaluation showed no evidence of tumor recurrence.

\section{DISCUSSION}

A fibrosarcoma is a tumor that arises from mesenchymal cells and is composed of malignant fibroblasts within a collagen background. When a fibrosarcoma is diagnosed during the early years of life, it is called a congenital infantile fibrosarcoma (IF) and represents 

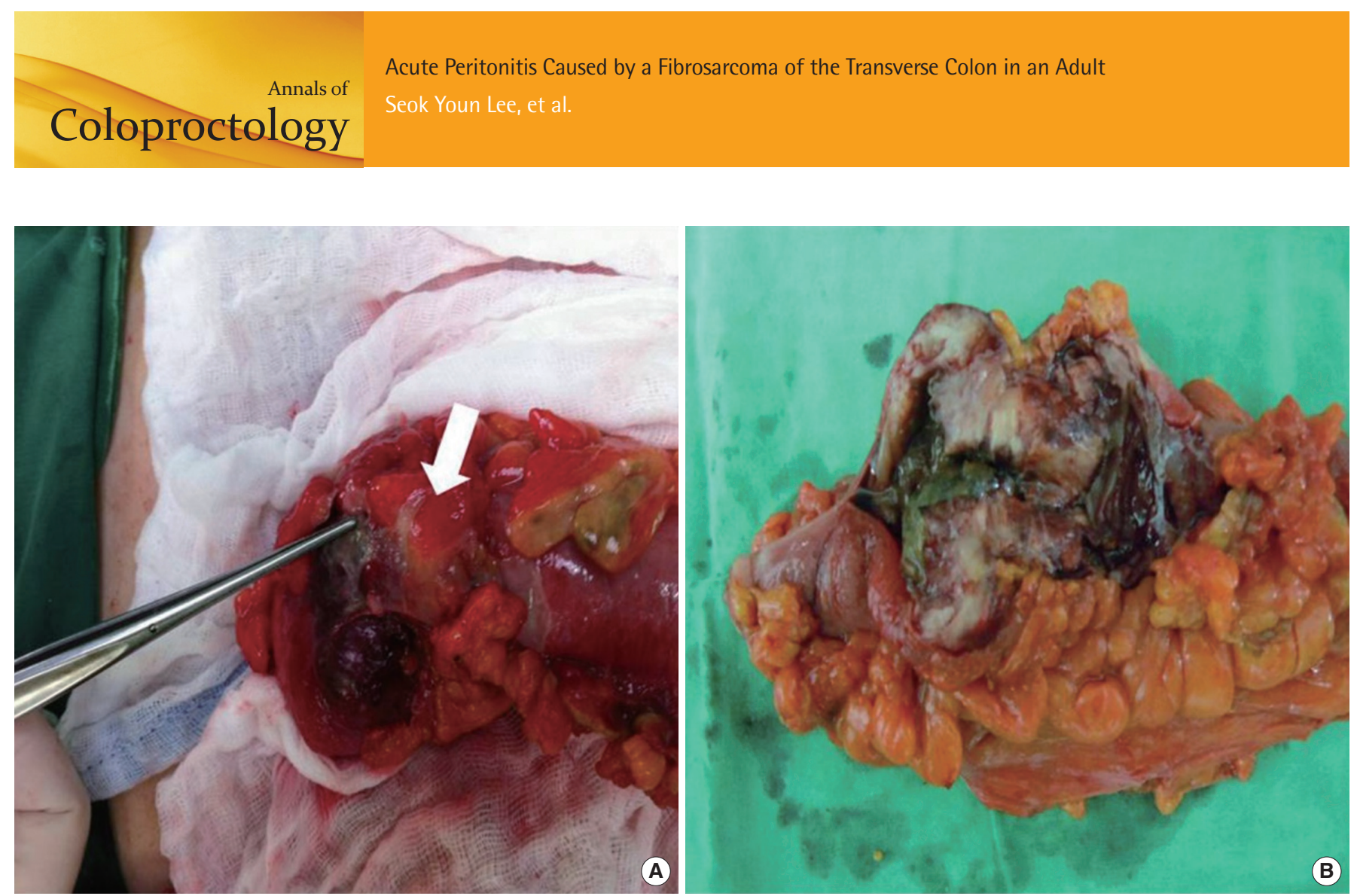

Fig. 3. (A, B) A gross, round mass was wrapped with the omentum around a distal part of the transverse colon (arrow).
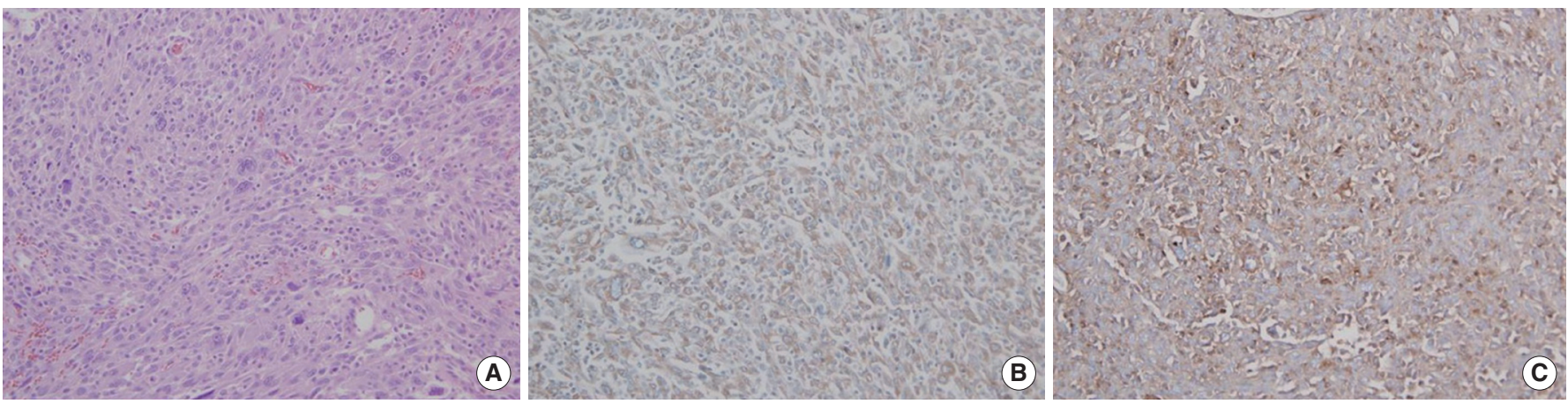

Fig. 4. Microscopic findings of the colon. Typical spindle cells that formed a fascicular arrangement with mitoses and a small amount of interstitial collagen $(\mathrm{H} \& \mathrm{E}, \times 200)$ are shown in panel A. Immunochemical stains of tumor cells that were positive for vimentin (vimentin, $\times 200)$ and negative for a1-antichymotrypsin (a1-antichymotrypsin, $\times 200)$ are shown in panels B and C, respectively.

less than $1 \%$ of all pediatric malignant tumors [5]. An adult fibrosarcoma, defined by the World Health Organization as a 'malignant neoplasm composed of fibroblasts with variable collagen production and, in classical cases, a "herringbone" architecture, is a very rare soft-tissue sarcoma [6]. Once considered the most common adult sarcoma, the incidence of adult fibrosarcoma has declined dramatically over the past several decades. This is due to the evolving classification of soft-tissue tumors and the recognition of clinically, genetically distinctive subtypes of fibrosarcomas. Also, the study of an adult fibrosarcoma has increased the understanding of many other mesenchymal and nonmesenchymal tumors that mimic fibrosarcomas. These include low-grade fibro- myxoid sarcomas, sclerosing epithelioid fibrosarcomas and fibrosarcomas arising from pre-existing dermatofibrosarcoma protuberans. However, a recent review of cases diagnosed as a fibrosarcoma at a single institution during a nearly 50 -year period showed that strictly-defined adult fibrosarcomas accounted for less than one percent of adult soft-tissue sarcomas [7].

A fibrosarcoma is usually located in subcutaneous tissues at various anatomic sites, most frequently involving the extremities and the axial regions. The existence of a fibrosarcoma in the colon is extremely rare. After a fibrosarcoma of the colon was first recognized in 1949, fibrosarcomas were associated with ulcerative colitis, which had generally been studied [1-4]. 
An adult fibrosarcoma is most commonly found in middle-aged and older adults (whose median age is 50 years). One recent series of studies reported adult fibrosarcomas to be more common in males [7]. The tumor usually occurs in deep soft tissues of the extremities and is often asymptomatic. In such cases, when anatomical amputation is not required, only a wide local excision is necessary. Visceral fibrosarcomas are not likely to exist, and most retroperitoneal fibrosarcomas represent low-grade dedifferentiated liposarcomas. In rare instances, for the fibrosarcomas in the gastrointestinal tract, diagnosis is often delayed because of the lack of specific presenting symptoms. Mainly, a fibrosarcoma presents as a mass either with or without pain. Its clinical presentations depend on the location and the extent of the mass. As presented in our case, the origin of the mass could not be identified preoperatively, but was confirmed by surgical exploration. Furthermore, in our case, a bowel perforation was associated with the fibrosarcoma of the colon, which had not been reported.

Due to its varied and nonspecific clinical presentation and the rarity of the condition, a fibrosarcoma of the colon is difficult to diagnose radiologically. The imaging modalities used are ultrasound, $\mathrm{CT}$, and magnetic resonance imaging (MRI). For tumors in the extremities and axial soft-tissue areas, MRI is thought to be superior to these other modalities. The masses appear well demarcated and have high signal intensity on T2 weighting [8].

By immunohistochemistry, a fibrosarcoma expresses vimentin and may occasionally show limited expression of SMA, representing myofibroblastic differentiation. CD34 positive tumors showing a fibrosarcoma-like morphology probably represent a fibrosarcoma arising in dermatofibrosarcoma protuberans. A low-grade fibromyxoid sarcoma shows a fibroblastic immunophenotype, expressing chiefly vimentin. Expression of epithelial membrane antigen (EMA) may be seen, potentially resulting in misdiagnosis as perineurioma. Expression of the MUC4 glycoprotein has been shown recently to be a highly sensitive and relatively specific marker of a low-grade fibromyxoid sarcoma. A sclerosing epithelioid fibrosarcoma typically express only vimentin, among conventional markers. MUC4 has been shown recently to be a sensitive marker of both a pure sclerosing epithelioid fibrosarcoma and those showing mixed features of a low-grade fibromyxoid sarcoma. Epithelial marker expression is rarely seen. Conventional dermatofibrosarcoma protuberans show diffuse expression of CD34 in nearly all cases and are typically negative for other markers, including cytokeratins, Factor XIIIa, S100 protein, EMA, actins and desmin. A wide variety of other malignant spindle cell neoplasms may potentially simulate fibrosarcomas, including malignant peripheral nerve sheath tumors, malignant solitary fibrous tumors, spindlecell variants of rhabdomyosarcomas, spindle-cell variants of angiosarcomas, and epithelioid sarcomas showing predominantly spindle morphologies. In superficial locations, spindle-cell malignant melanomas and sarcomatoid carcinomas must also be excluded. In general, a limited panel of immunostains, to include some combination of those recognizing cytokeratins, S100 protein, CD34,
SMA, desmin, myogenin, CD31, should allow these distinctions in most instances [9].

Surgical excision remains the principle of treatment, showing a good result when a complete resection with tumor-free margins is achieved. In some recent studies of strictly-defined adult fibrosarcomas, as reported by Bahrami and Folpe [7], over $80 \%$ of tumors were high-grade, with one of the four low-grade lesions progressing to a high-grade sarcoma in a local recurrence. These fibrosarcomas were aggressive, with multiple local recurrences in the lymph nodes and parenchymal metastases, and, at five years, the overall survival was less than $55 \%$. Due to the relatively small number of fibrosarcoma cases, no correlation could be made between clinicopathological variables and survival outcome [7].

In contrast to an adult fibrosarcoma, an IF rarely exhibits a distant metastasis and has a good prognosis; greater than $90 \%$ of patients may be cured with appropriate management [10]. There is no defined role for adjuvant chemotherapy or radiotherapy after complete surgical excision of the tumor [11]. Usually, a patient who is diagnosed as having high-risk stage II colon cancer should receive adjuvant chemotherapy. However, for a patient with a perforated colonic fibrosarcoma, there is no evidence that chemotherapy will help the patient. If the cancer recurs, our concern is how we can treat the patient. More research on gastrointestinal fibrosarcomas is not needed. In our patient, during a follow-up period, we could not identify any evidence of local recurrence or distant metastasis.

\section{CONFLICTS OF INTEREST}

No potential conflict of interest relevant to this article was reported.

\section{ACKNOWLEDGMENTS}

This paper was supported by Wonkwang University in 2012.

\section{REFERENCES}

1. Bassler A, Peters AG. Fibrosarcoma, an unusual complication of ulcerative colitis; report of a case. Arch Surg 1949;59:227-31.

2. Simon R, Blondet P, Lecoeur D. Fibrosarcoma of the left angle of the colon in a young girl. Mem Acad Chir (Paris) 1964;90:311-5.

3. Hoehn JL, Hamilton GH, Beltaos E. Fibrosarcoma of the colon. J Surg Oncol 1980;13:223-5.

4. Bonser RS, McMaster P, Acland PR, Parratt J. Fibrosarcoma of the transverse colon. J Surg Oncol 1986;31:34-5.

5. Chung EB, Enzinger FM. Infantile fibrosarcoma. Cancer 1976; 38:729-39.

6. Fletcher CD, Bridge JA, Hogendoorn PC, Mertens F. WHO classification of tumours of soft tissue and bone. 4th ed. Lyons: IARC Press; 2013.

7. Bahrami A, Folpe AL. Adult-type fibrosarcoma: a reevaluation of 
163 putative cases diagnosed at a single institution over a 48 -year period. Am J Surg Pathol 2010;34:1504-13.

8. Lee MJ, Cairns RA, Munk PL, Poon PY. Congenital-infantile fibrosarcoma: magnetic resonance imaging findings. Can Assoc Radiol J 1996;47:121-5.

9. Folpe AL. Fibrosarcoma: a review and update. Histopathology 2014;64:12-25.
10. Pritchard DJ, Soule EH, Taylor WF, Ivins JC. Fibrosarcoma: a clinicopathologic and statistical study of 199 tumors of the soft tissues of the extremities and trunk. Cancer 1974;33:888-97.

11. Orbach D, Rey A, Cecchetto G, Oberlin O, Casanova M, Thebaud E, et al. Infantile fibrosarcoma: management based on the European experience. J Clin Oncol 2010;28:318-23. 